\title{
Improved lower bounds on sizes of single-error correcting codes
}

\author{
Simon Litsyn • Beniamin Mounits
}

Received: 16 January 2006 / Revised: 18 August 2006 /

Accepted: 25 August 2006 / Published online: 1 November 2006

(C) Springer Science+Business Media, LLC 2006

\begin{abstract}
A construction of codes of length $n=q+1$ and minimum Hamming distance 3 over $G F(q) \backslash\{0\}$ is given. Substitution of the derived codes into a concatenation construction yields nonlinear binary single-error correcting codes with better than known parameters. In particular, new binary single-error correcting codes having more codewords than the best previously known in the range $n \leq 512$ are obtained for the lengths 64-66, 128-133, 256-262, and 512.
\end{abstract}

Keywords $A(n, d) \cdot$ MDS code $\cdot$ Weight distribution

\section{AMS Classification 94B25}

\section{Introduction}

Let $(n, M, d)_{q}$ denote a code of length $n$, minimum Hamming distance $d$ and cardinality $M$ over field $G F(q)$, whereas $[n, k, d]_{q}$ is a linear $\left(n, q^{k}, d\right)_{q}$ code. In a binary case we will omit the lower index and write $(n, M, d)$. Let $A(n, d)$ denote the maximum number of codewords in a binary code of length $n$ and minimum Hamming distance $d$. The quantity $A(n, d)$ is of basic interest in coding theory. Lower bounds on $A(n, d)$ are obtained by constructions. For a survey on the known lower bounds the reader is referred to [4].

Communicated by R. Hill.

\section{S. Litsyn}

Department of Electrical Engineering, Tel Aviv University, Tel Aviv, Israel

e-mail: litsyn@eng.tau.ac.il

B. Mounits $(\bowtie)$

CWI, Amsterdam, The Netherlands

e-mail: B.Mounits@cwi.nl 
In this correspondence we consider lower bounds on $A(n, 3)$. One of the most powerful tools in obtaining good lower bounds on $A(n, 3)$ is the following method which consists of two steps:

- Subalphabet subcode construction: Suppose we have a nonbinary $(n, M, d)_{Q}$ code $\mathcal{C}$ over an alphabet $\mathcal{A}=\left\{\alpha_{1}, \alpha_{2}, \ldots, \alpha_{Q}\right\}$. Then, for $\mathcal{S} \subseteq \mathcal{A}$ we can construct $\left(n, M^{\prime}, d^{\prime}\right)_{|\mathcal{S}|}$ subcode $\mathcal{C}^{\prime}$ of $\mathcal{C}$ over subalphabet $\mathcal{S}$ of $\mathcal{A}$, i.e., $\mathcal{C}^{\prime}$ consists of those codewords of $\mathcal{C}$ which have values from $\mathcal{S}$ in all the coordinates. It is clear that $M^{\prime} \leq M$ and $d^{\prime} \geq d$.

- Concatenation construction: Each coordinate value $\alpha_{i} \in \mathcal{S}$ is substituted by codewords of a binary code $\mathcal{C}_{i}$ with parameters $\left(n_{0}, M_{i}, d\right)$, such that $\mathcal{C}_{i} \cap \mathcal{C}_{j}=\emptyset$ for $i \neq j$. Thus, the resulting binary code has length $n \cdot n_{0}$ and minimum distance $d$. The size of the code obtained depends on the values $M_{i}, 1 \leq i \leq|\mathcal{S}|$.

To obtain good binary codes with minimum distance 3 , one usually takes $|\mathcal{S}|=2^{m}$ and codes $\mathcal{C}_{i}$ be cosets of the binary Hamming code of length $2^{m}-1$. For a description of this method and some related constructions the reader is referred to $[1-4,6]$.

In Sect. 2, we present a new construction which is a modification of the method given above. We construct a subalphabet subcode such that the alphabet sizes of the coordinates of the new code are not all equal $|\mathcal{S}|$. In Sect. 3, we apply the new construction and obtain improved lower bounds on $A(n, 3)$.

The following notations will be used. The binary Hamming code of order $s, \mathcal{H}_{1}(s)$, is a $\left(2^{s}-1,2^{2^{s}-1-s}, 3\right)$ code. Given $s,\left\{\mathcal{H}_{i}(s): 1 \leq i \leq 2^{s}\right\}$ denotes the collection of non-intersecting codes consisting of the binary Hamming code and its $2^{s}-1$ cosets.

\section{Construction}

For $\mathcal{C}$ being an $(n, M, d)$ code, let $A_{w}$ be the number of codewords of weight $w$. The numbers $A_{0}, A_{1}, \ldots, A_{n}$ are called the weight distribution of $C$. Clearly $A_{0}+A_{1}+$ $\cdots+A_{n}=M$.

Throughout $\mathcal{C}$ will denote the nonbinary MDS Hamming code having parameters $[n=q+1, k=q-1,3]_{q}$ over $G F(q)$. Let $G F(q)=\left\{0, \alpha_{1}, \ldots, \alpha_{i}, \ldots, \alpha_{q-1}\right\}$. The weight distribution of MDS codes is known (see, e.g. [5, pp. 320-321]).

Theorem 1 The number of codewords of weight $w$ in an $[n, k, d=n-k+1]_{q} M D S$ code over $G F(q)$ is

$$
A_{w}=\left(\begin{array}{l}
n \\
w
\end{array}\right)(q-1) \sum_{j=0}^{w-d}(-1)^{j}\left(\begin{array}{c}
w-1 \\
j
\end{array}\right) q^{w-d-j}
$$

We denote $\mathcal{C}_{w}=\{c \in \mathcal{C}: w t(c)=w\}$. Obviously $\mathcal{C}=\bigcup_{w=0}^{n} \mathcal{C}_{w}$ and $\left|\mathcal{C}_{w}\right|=A_{w}$

Lemma 1 For every coordinate and any $i \in\{1, \ldots, q-1\}$, the number of codewords of $\mathcal{C}_{q+1}$ having $\alpha_{i}$ in this coordinate is $\frac{A_{q+1}}{q-1}$. 
Proof Denote

$$
B_{\ell}^{j}=\left\{u=\left(u_{1}, \ldots, u_{k}\right) \in G F(q)^{k}: u G=c \in \mathcal{C}_{q+1}, c_{\ell}=\alpha_{j} \in G F(q) \backslash\{0\}\right\},
$$

where $G$ is a generator matrix of $\mathcal{C}$ and $c_{\ell}$ is the $\ell$ th coordinate of the codeword $c$. We wish to prove that for each $\ell, 1 \leq \ell \leq q+1$, and for any $i, j \in\{1, \ldots, q-1\}$, $\left|B_{\ell}^{j}\right|=\left|B_{\ell}^{i}\right|$. Note that $u \in B_{\ell}^{j}$ if and only if

$$
\alpha_{i} \cdot \alpha_{j}^{-1} u=\left(\alpha_{i} \cdot \alpha_{j}^{-1} u_{1}, \ldots, \alpha_{i} \cdot \alpha_{j}^{-1} u_{k}\right) \in B_{\ell}^{i},
$$

where $\alpha_{j}^{-1}$ denotes multiplicative inverse of $\alpha_{j}$ in $G F(q)$, which completes the proof.

Lemma 2 For every coordinate, the number of codewords of $\mathcal{C}_{q}$ having 0 in that coordinate is $A_{q} /(q+1)$.

Proof Let

$$
H=\left[h_{1}, h_{2}, \ldots, h_{\ell}, \ldots, h_{n}\right]
$$

be a parity check matrix of $\mathcal{C}$ and

$$
H_{\ell}^{\prime}=\left[h_{1}, h_{2}, \ldots, h_{\ell-1}, h_{\ell+1} \ldots, h_{n}\right]
$$

be a parity check matrix of the $\operatorname{code} \mathcal{C}^{\prime}$ which is obtained by deleting the $\ell$ th column $h_{\ell}$ from $H$. Since $\mathcal{C}$ is MDS, it follows from [5, Corollary 3, p.319] that every $n-k=$ $q+1-(q-1)=2$ columns of $H$ are linearly independent. Thus every two columns of $H_{\ell}^{\prime}$ are also linearly independent, and by the same corollary [5, Corollary 3, p.319], the code $\mathcal{C}^{\prime}$ having parameters $\left[n^{\prime}=n-1=q, k^{\prime}=k-1=q-2, d=n^{\prime}-k^{\prime}+1=3\right]_{q}$ is MDS. Obviously

$$
\mathcal{C}_{q}^{\prime}=\left\{\left(c_{1}, \ldots, c_{\ell-1}, c_{\ell+1}, \ldots, c_{n}\right):\left(c_{1}, \ldots, c_{\ell-1}, 0, c_{\ell+1}, \ldots, c_{n}\right) \in \mathcal{C}_{q}\right\} .
$$

Using (1) we have

$$
\begin{aligned}
A_{q}=\left|\mathcal{C}_{q}\right| & =(q+1)(q-1) \sum_{j=0}^{q-d}(-1)^{j}\left(\begin{array}{c}
q-1 \\
j
\end{array}\right) q^{q-d-j}, \\
\left|\mathcal{C}_{q}^{\prime}\right| & =(q-1) \sum_{j=0}^{q-d}(-1)^{j}\left(\begin{array}{c}
q-1 \\
j
\end{array}\right) q^{q-d-j}
\end{aligned}
$$

and therefore

$$
\left|\mathcal{C}_{q}^{\prime}\right|=\frac{A_{q}}{q+1} .
$$

Lemma 3 For $a[q+1, q-1,3]_{q} M D S$ code $\mathcal{C}, q$ odd,

$$
A_{q+1}=\frac{q-1}{q^{2}}\left((q-1)^{q}-q^{2}+1\right) .
$$


Proof Using (1), we obtain

$$
\begin{aligned}
A_{q+1}= & (q-1) \sum_{j=0}^{q-2}(-1)^{j}\left(\begin{array}{c}
q \\
j
\end{array}\right) q^{q-2-j}=\frac{q-1}{q^{2}} \sum_{j=0}^{q-2}(-1)^{j}\left(\begin{array}{c}
q \\
j
\end{array}\right) q^{q-j} \\
& =\frac{q-1}{q^{2}}\left(\sum_{j=0}^{q}(-1)^{j}\left(\begin{array}{c}
q \\
j
\end{array}\right) q^{q-j}-\sum_{j=q-1}^{q}(-1)^{j}\left(\begin{array}{c}
q \\
j
\end{array}\right) q^{q-j}\right)=\frac{q-1}{q^{2}}\left((q-1)^{q}-q^{2}+1\right) .
\end{aligned}
$$

Lemma 4 For $a[q+1, q-1,3]_{q} \operatorname{MDS} \operatorname{code} \mathcal{C}, q$ odd,

$$
A_{q}=\frac{q+1}{q^{2}}\left((q-1)^{q}+(q-1)\left(q^{2}-q-1\right)\right) .
$$

Proof Using (1), we obtain

$$
\begin{aligned}
A_{q}= & (q+1)(q-1) \sum_{j=0}^{q-3}(-1)^{j}\left(\begin{array}{c}
q-1 \\
j
\end{array}\right) q^{q-3-j}=\frac{q^{2}-1}{q^{2}} \sum_{j=0}^{q-3}(-1)^{j}\left(\begin{array}{c}
q-1 \\
j
\end{array}\right) q^{q-1-j} \\
& =\frac{q^{2}-1}{q^{2}}\left(\sum_{j=0}^{q-1}(-1)^{j}\left(\begin{array}{c}
q-1 \\
j
\end{array}\right) q^{q-1-j}-\sum_{j=q-2}^{q-1}(-1)^{j}\left(\begin{array}{c}
q-1 \\
j
\end{array}\right) q^{q-1-j}\right) \\
& =\frac{q^{2}-1}{q^{2}}\left((q-1)^{q-1}+q^{2}-q-1\right)=\frac{q+1}{q^{2}}\left((q-1)^{q}+(q-1)\left(q^{2}-q-1\right)\right) .
\end{aligned}
$$

Let $m \in\{1, \ldots, q-2\}$. We take $m \cdot \frac{A_{q+1}}{q-1}$ codewords of $\mathcal{C}_{q+1}$ having $\alpha_{1}, \ldots, \alpha_{m}$ in the $\ell$ th coordinate, and $\frac{A_{q}}{q+1}$ codewords of the code $\mathcal{C}_{q}$ having 0 at the $\ell$ th coordinate which we substitute by $\alpha_{m+1}$. Therefore, we obtain a $\left(q+1, m \frac{A_{q+1}}{q-1}+\frac{A_{q}}{q+1}, 3\right)_{q-1}$ code over $G F(q) \backslash\{0\}$. Let us denote this code by $\mathcal{D}(m, q)$. If $q$ is odd it is easy to evaluate, using (2) and (3), that $\mathcal{D}(m, q)$ has parameters

$$
\left(q+1, \frac{(m+1)(q-1)^{q}+(q-1)\left(q^{2}-q-1\right)-m\left(q^{2}-1\right)}{q^{2}}, 3\right)_{q-1} .
$$

Now, let us consider the case $m=2^{s}-1$ and $q=2^{t}+1$. We plug in the values for $m$ and $q$ and obtain that $\mathcal{D}\left(2^{s}-1,2^{t}+1\right)$ is an $(n, M, 3)_{2^{t}}$ code, where

$$
n=2^{t}+2, \quad M=\frac{2^{t 2^{t}+t+s}+2^{3 t}+2^{2 t+1}+2^{t}-2^{2 t+s}-2^{t+s+1}}{2^{2 t}+2^{t+1}+1} .
$$

We know that in the code $\mathcal{D}\left(2^{s}-1,2^{t}+1\right)$, in the $\ell$ th coordinate, only $2^{s}$ symbols can appear from the $2^{t}$ symbols of $G F\left(2^{t}+1\right) \backslash\{0\}$. Therefore, we can encode the codewords of $\mathcal{D}\left(2^{s}-1,2^{t}+1\right)$ in the following way to obtain a binary code.

In the $\ell$-th coordinate: If $s=1$, then $\alpha_{1} \rightarrow 0$ and $\alpha_{2} \rightarrow 1$. If $s \geq 2$, then

$$
\begin{gathered}
\alpha_{1} \rightarrow \text { all the codewords of } \mathcal{H}_{1}(s), \\
\alpha_{2} \rightarrow \text { all the codewords of } \mathcal{H}_{2}(s), \\
\vdots \\
\alpha_{i} \rightarrow \text { all the codewords of } \mathcal{H}_{i}(s),
\end{gathered}
$$




$$
\alpha_{2^{s}} \rightarrow \text { all the codewords of } \mathcal{H}_{2^{s}}(s) .
$$

In the rest of coordinates: We encode using the following rules

$$
\begin{gathered}
\alpha_{1} \rightarrow \text { all the codewords of } \mathcal{H}_{1}(t), \\
\alpha_{2} \rightarrow \text { all the codewords of } \mathcal{H}_{2}(t), \\
\vdots \\
\alpha_{i} \rightarrow \text { all the codewords of } \mathcal{H}_{i}(t), \\
\vdots \\
\alpha_{2^{t}} \rightarrow \text { all the codewords of } \mathcal{H}_{2^{t}}(t) .
\end{gathered}
$$

By this encoding the code $\mathcal{D}\left(2^{s}-1,2^{t}+1\right)$ transforms into a binary code, that will be denoted by $\mathcal{B}(s, t)$, having parameters $(n, M, 3)$, where

$n=2^{2 t}+2^{s}-2, \quad M=\frac{2^{t 2^{t}+t+s}+2^{3 t}+2^{2 t+1}+2^{t}-2^{2 t+s}-2^{t+s+1}}{2^{2 t}+2^{t+1}+1} 2^{2^{2 t}+2^{s}-t 2^{t}-t-s-2}$.

Therefore, we have proved the following theorem.

Theorem 2 Let $q$ be a prime power of the form $q=2^{t}+1$, and $m$ be an integer of the form $m=2^{s}-1, s \leq t$. There exists a binary $(n, M, 3)$ code $\mathcal{B}(s, t)$, where

$n=2^{2 t}+2^{s}-2, \quad M=\frac{2^{t 2^{t}+t+s}+2^{3 t}+2^{2 t+1}+2^{t}-2^{2 t+s}-2^{t+s+1}}{2^{2 t}+2^{t+1}+1} 2^{2^{2 t}+2^{s}-t 2^{t}-t-s-2}$.

\section{Improved lower bounds on $A(n, 3)$ for $n \leq 512$}

Here we apply the construction from the previous section to improve on the best known values of $A(n, 3)$ for $n \leq 512$. The following table presents these improvements. Codes that are obtained by shortening and having the same redundancy do not appear in the table.

\begin{tabular}{llll}
\hline Length & $\mathcal{B}(s, t)$ & $|\mathcal{B}(s, t)|$ & Previous bound \\
\hline 64 & $\mathcal{B}(1,3)$ & $1657012 \times 2^{37}$ & $1657009 \times 2^{37}$ \\
66 & $\mathcal{B}(2,3)$ & $1657010 \times 2^{39}$ & $1657009 \times 2^{39}$ \\
256 & $\mathcal{B}(1,4)$ & $1021273028302258920 \times 2^{188}$ & $1021273028302258913 \times 2^{188}$ \\
258 & $\mathcal{B}(2,4)$ & $1021273028302258916 \times 2^{190}$ & $1021273028302258913 \times 2^{190}$ \\
262 & $\mathcal{B}(3,4)$ & $1021273028302258914 \times 2^{194}$ & $1021273028302258913 \times 2^{194}$ \\
\hline
\end{tabular}

By using the $(u, u+v)$ construction $[5, \mathrm{p} .76]$ on the new codes of lengths $64-66$ and 256, we obtain codes of lengths 128-133 and 512 in the range $n \leq 512$, that improve on the best known values.

\begin{tabular}{lll}
\hline Length & New bound & Previous bound \\
\hline 128 & $1657012 \times 2^{100}$ & $1657009 \times 2^{100}$ \\
133 & $1657010 \times 2^{105}$ & $1657009 \times 2^{105}$ \\
512 & $1021273028302258920 \times 2^{443}$ & $1021273028302258913 \times 2^{443}$ \\
\hline
\end{tabular}


Acknowledgments The authors wish to thank Tuvi Etzion for many helpful discussions and two anonymous referees for their constructive comments. This research was supported in part by grant ISF551-03.

\section{References}

1. Hämäläinen HO (1988) Two new binary codes with minimum distance three. IEEE Trans Inform Theory 34(4):875

2. Kabatyanskii GA, Panchenko VI (1988) Packings and coverings of the Hamming space by sphers of radius one. Problems Inform Trans 24(4):3-16

3. Kalbfleisch JG, Stanton RG, Horton JD (1971) On covering sets and error-correcting codes. J Comb Theory 11:233-250

4. Litsyn S (1998) An updated table of the best binary codes known. In: Pless VS, Huffman WC (eds) Handbook of Coding Theory vol. 1. Elsevier, Amsterdam, pp 463-498

5. MacWilliams FJ, Sloane NJA (1977) The theory of error-correcting codes. North-Holland, Amsterdam

6. Östergård PRJ, Kaikkonen MK (1996) New single-error-correcting codes. IEEE Trans Inform Theory 42(4):pp 1261-1262 